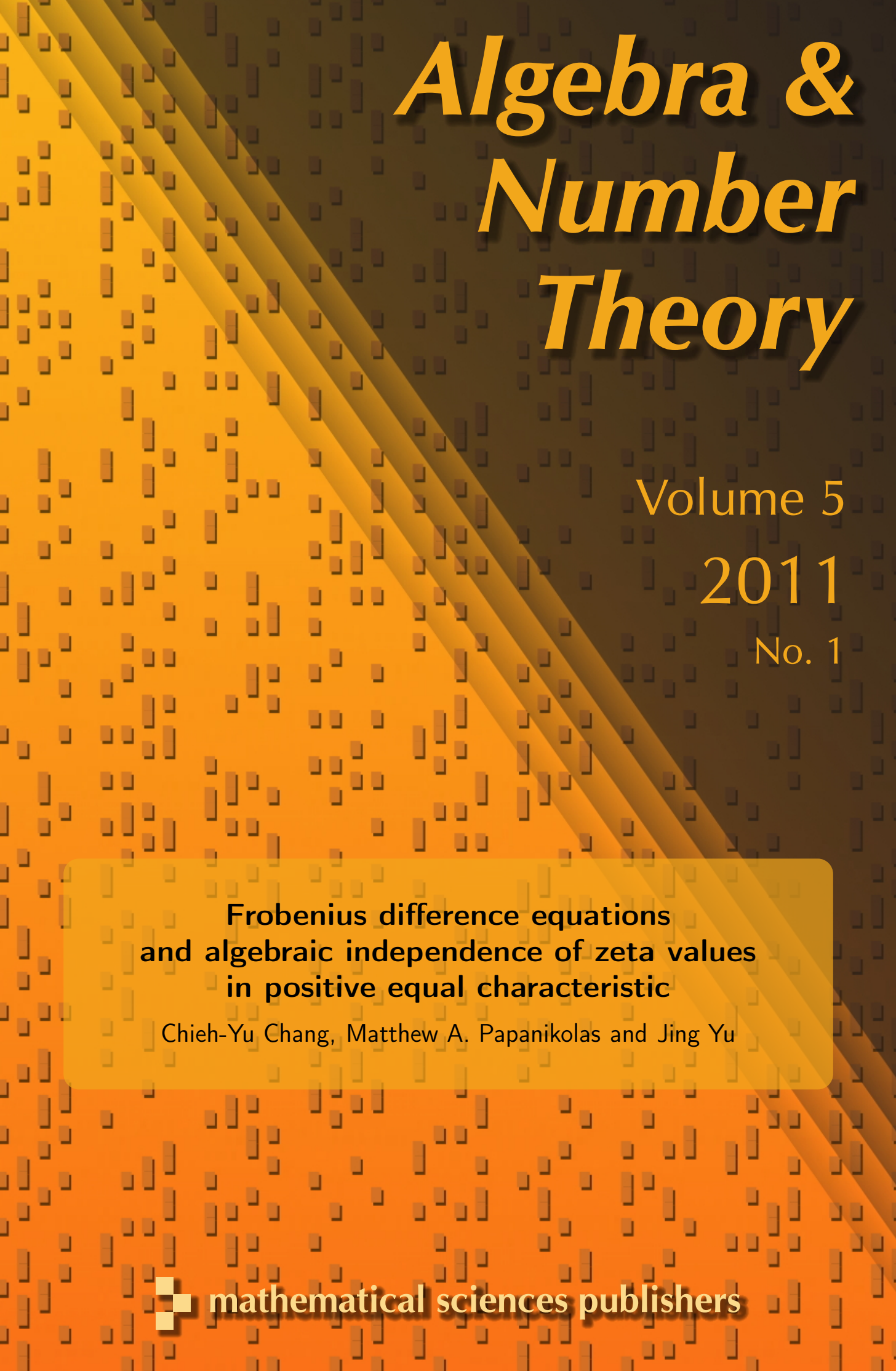

Frobenius difference equations and algebraic independence of zeta values in positive equal characteristic

Chieh-Yu Chang, Matthew A. Papanikolas and Jing Yu \lrcorner 


\title{
Frobenius difference equations and algebraic independence of zeta values in positive equal characteristic
}

\author{
Chieh-Yu Chang, Matthew A. Papanikolas and Jing Yu
}

\begin{abstract}
By analogy with the Riemann zeta function at positive integers, for each finite field $\mathbb{F}_{p^{r}}$ with fixed characteristic $p$, we consider Carlitz zeta values $\zeta_{r}(n)$ at positive integers $n$. Our theorem asserts that among the zeta values in the set $\bigcup_{r=1}^{\infty}\left\{\zeta_{r}(1), \zeta_{r}(2), \zeta_{r}(3), \ldots\right\}$, all the algebraic relations are those relations within each individual family $\left\{\zeta_{r}(1), \zeta_{r}(2), \zeta_{r}(3), \ldots\right\}$. These are the algebraic relations coming from the Euler-Carlitz and Frobenius relations. To prove this, a motivic method for extracting algebraic independence results from systems of Frobenius difference equations is developed.
\end{abstract}

\section{Introduction}

1.1. Motivic transcendence theory. Classically, Grothendieck's period conjecture for abelian varieties predicts that the dimension of the Mumford-Tate group of an abelian variety over $\overline{\mathbb{Q}}$ should be equal to the transcendence degree of the field generated by its period matrix over $\overline{\mathbb{Q}}$. Conjecturally, the Mumford-Tate group is the motivic Galois group from Tannakian duality, and therefore Grothendieck's conjecture provides an interpretation of the algebraic relations among periods in question by way of motivic Galois groups.

We are concerned with the algebraic independence of special zeta values over function fields with varying finite constant fields in positive equal characteristic. In the positive characteristic world, there is the concept of $t$-motives introduced by Anderson [1986], dual to the concept of $t$-modules. The third author showed that the structure of $t$-modules is key for proving many interesting linear independence results about special values in this setting [Yu 1997]. The breakthrough in passing from linear independence to algebraic independence by way of $t$-motives began

The first author was supported by NSC and NCTS. The second author was supported by NSF Grants DMS-0600826 and DMS-0903838. The third author was supported by NSC Grant No. 99-2119-M002-024.

MSC2000: primary 11J93; secondary 11M38, $11 \mathrm{G} 09$.

Keywords: Algebraic independence, Frobenius difference equations, $t$-motives, zeta values. 
with Anderson, Brownawell and the second author, and in particular with the linear independence criterion of [Anderson et al. 2004] (the ABP criterion).

By introducing a Tannakian formalism for rigid analytically trivial pre- $t$-motives and relating it to the Galois theory of Frobenius difference equations, the second author [Papanikolas 2008] has shown that the Galois group of a rigid analytically trivial pre- $t$-motive is isomorphic to its difference Galois group. Furthermore, the second author has successfully used the ABP criterion to show that the transcendence degree of the field generated by the period matrix of an ABP motive (that is, the pre-t-motive comes from a uniformizable abelian $t$-module) is equal to the dimension of its Galois group. More generally, we say that a rigid analytically trivial pre- $t$-motive has the GP (Grothendieck period) property if the transcendence degree of the field generated by its period matrix is equal to the dimension of its Galois group (for more details of terminology, see Section 2).

Using a refined version of the ABP criterion proved by the first author [Chang 2009], we observe that there are many pre-t-motives that are not ABP motives but that have the GP property. This motivates us to introduce a method of uniformizing the Frobenius twisting operators with respect to different constant fields for those pre- $t$-motives that have the GP property. The pre- $t$-motive we obtain in this way is defined over a larger constant field, but still has the GP property (see Corollary 2.2.4). This technique is very useful when dealing with the problem of determining all the algebraic relations among various special values of arithmetic interest in a fixed positive characteristic. It is used in this paper to study special zeta values. For another application to special arithmetic gamma values, see [Chang et al. 2010].

1.2. Carlitz zeta values. Let $p$ be a prime, and let $\mathbb{F}_{p^{r}}[\theta]$ be the polynomial ring in $\theta$ over the finite field $\mathbb{F}_{p^{r}}$ of $p^{r}$ elements. Our aim is to determine all the algebraic relations among the zeta values

$$
\zeta_{r}(n):=\sum_{\substack{a \in \mathbb{F}_{p^{r}}[\theta] \\ a \text { monic }}} \frac{1}{a^{n}} \in \mathbb{F}_{p^{r}}((1 / \theta)) \subseteq \overline{\mathbb{F}_{p}}((1 / \theta)),
$$

where $r$ and $n$ vary over all positive integers. Each $\zeta_{r}(n)$ lies in $\mathbb{F}_{p}((1 / \theta))$, since it is fixed by the automorphism $\left(\sum a_{i}(1 / \theta)^{i} \mapsto \sum a_{i}^{p}(1 / \theta)^{i}\right): \overline{\mathbb{F}_{p}}((1 / \theta)) \rightarrow \overline{\mathbb{F}_{p}}((1 / \theta))$.

The study of these zeta values was begun by Carlitz [1935]. For a fixed positive integer $r$, he discovered that there is a constant $\tilde{\pi}_{r}$, algebraic over $\mathbb{F}_{p^{r}}((1 / \theta))$, such that $\zeta_{r}(n) / \tilde{\pi}_{r}^{n}$ lies in $\mathbb{F}_{p}(\theta)$ if $n$ is divisible by $p^{r}-1$. The quantity $\tilde{\pi}_{r}$ arises as a fundamental period of the Carlitz $\mathbb{F}_{p^{r}}[t]$-module $C_{r}$, and Wade [1941] showed that $\tilde{\pi}_{r}$ is transcendental over $\mathbb{F}_{p}(\theta)$.

We say that a positive integer $n$ is $(p, r)$-even if it is a multiple of $p^{r}-1$. Thus the situation of Carlitz zeta values at $(p, r)$-even positive integers is completely 
analogous to that of the Riemann zeta function at even positive integers. For these $(p, r)$-even $n$, we call the $\mathbb{F}_{p}(\theta)$-linear relations between $\zeta_{r}(n)$ and $\tilde{\pi}_{r}^{n}$ the EulerCarlitz relations. Because the characteristic is positive, there are also Frobenius $p$-th power relations among these zeta values: for positive integers $m, n$,

$$
\zeta_{r}\left(p^{m} n\right)=\zeta_{r}(n)^{p^{m}}
$$

Anderson and Thakur [1990] and Yu [1991; 1997] made several breakthroughs in understanding Carlitz zeta values. Using the $t$-module method, the transcendence of $\zeta_{r}(n)$ for all positive integers $n$, and in particular for odd $n$ (that is, $n$ not divisible by $\left.p^{r}-1\right)$, was proved, and it was also proved that the Euler-Carlitz relations are the only $\overline{\mathbb{F}_{p}(\theta)}$-linear relations among $\left\{\zeta_{r}(n), \tilde{\pi}_{r}^{m} ; m, n \in \mathbb{N}\right\}$. In [Chang and Yu 2007], the first and third authors used ABP motives instead of $t$-modules to show that for fixed $r$, the Euler-Carlitz relations and the Frobenius $p$-th power relations account for all the algebraic relations over $\overline{\mathbb{F}_{p}(\theta)}$ among the Carlitz zeta values

$$
\tilde{\pi}_{r}, \zeta_{r}(1), \zeta_{r}(2), \zeta_{r}(3), \ldots
$$

To complete the story of Carlitz zeta values, the next natural question is what happens if $r$ varies. Denis [1998] proved the algebraic independence of all fundamental periods $\left\{\tilde{\pi}_{1}, \tilde{\pi}_{2}, \tilde{\pi}_{3}, \ldots\right\}$ as the constant field varies. Thus, in view of [Chang and Yu 2007], one expects that for the bigger set of zeta values,

$$
\bigcup_{r=1}^{\infty}\left\{\zeta_{r}(1), \zeta_{r}(2), \zeta_{r}(3), \ldots\right\}
$$

the Euler-Carlitz relations and the Frobenius $p$-th power relations still account for all the algebraic relations. This is indeed the case, as we find from the following theorem (stated subsequently as Corollary 4.5.2).

Theorem 1.2.1. Given any positive integers s and $d$, the transcendence degree of the field

$$
\overline{\mathbb{F}_{p}(\theta)}\left(\bigcup_{r=1}^{d}\left\{\tilde{\pi}_{r}, \zeta_{r}(1), \ldots, \zeta_{r}(s)\right\}\right)
$$

over $\overline{\mathbb{F}_{p}(\theta)}$ is

$$
\sum_{r=1}^{d}\left(s-\left\lfloor\frac{s}{p}\right\rfloor-\left\lfloor\frac{s}{p^{r}-1}\right\rfloor+\left\lfloor\frac{s}{p\left(p^{r}-1\right)}\right\rfloor+1\right) .
$$

1.3. Outline. Our strategy is to construct a pre-t-motive that has the GP property and whose period matrix accounts for the Carlitz zeta values in question. In [Chang and Yu 2007], an ABP motive has already been constructed for Carlitz zeta values with respect to a fixed constant field. The problem here is one concerning varying the constant fields in a fixed characteristic, and one has to uniformize Frobenius powers in order to apply the method developed in [Papanikolas 2008]. 
This paper is organized as follows. In Section 2, we review Papanikolas' theory and investigate the pre-t-motives that have the GP property. Here we introduce the mechanism of uniformizing Frobenius twisting operators while taking direct sums. Section 3 includes discussions about rigid analytically trivial pre- $t$-motives of type $\mathbf{S V}$, that is, whose Galois groups are extensions of split tori by vector groups. The heart of this section is Theorem 3.2.2, where we determine the dimensions of Galois groups of direct sums of pre-t-motives of type $\mathbf{S V}$. The pre- $t$-motive for Theorem 1.2.1 is constructed in Section 4, and we prove that it satisfies the conditions of Theorem 3.2.2. Finally, we calculate its dimension explicitly in Theorem 4.5.1, which then has Theorem 1.2.1 as direct consequence.

\section{2. $t$-motivic Galois groups}

\subsection{Notation.}

\subsubsection{Table of symbols.}

$\mathbb{F}_{p}:=$ the finite field of $p$ elements, $p$ a prime number.

$k:=\mathbb{F}_{p}(\theta):=$ the rational function field in the variable $\theta$ over $\mathbb{F}_{p}$.

$k_{\infty}:=\mathbb{F}_{p}((1 / \theta))$, completion of $k$ with respect to the infinite place.

$\overline{k_{\infty}}:=$ a fixed algebraic closure of $k_{\infty}$.

$\bar{k}:=$ the algebraic closure of $k$ in $\overline{k_{\infty}}$.

$\mathbb{C}_{\infty}:=$ completion of $\overline{k_{\infty}}$ with respect to the canonical extension of the infinite place.

$|\cdot|_{\infty}:=$ a fixed absolute value for the completed field $\mathbb{C}_{\infty}$ with $|\theta|_{\infty}=p$.

$\mathbb{C}_{\infty} \llbracket t \rrbracket:=$ the ring of power series in the variable $t$ over $\mathbb{C}_{\infty}$.

$\mathbb{C}_{\infty}((t)):=$ the field of Laurent series in the variable $t$ over $\mathbb{C}_{\infty}$.

$\mathbb{T}:=\left\{f \in \mathbb{C}_{\infty} \llbracket t \rrbracket \mid f\right.$ converges on $\left.|t|_{\infty} \leq 1\right\}$. This is known as the Tate algebra.

$\mathbb{L}:=$ the fraction field of $\mathbb{\mathbb { T }}$.

$\sigma:=\sigma_{p}:=\left(\sum_{i} a_{i} t^{i} \mapsto \sum_{i} a_{i}^{1 / p} t^{i}\right): \mathbb{C}_{\infty}((t)) \rightarrow \mathbb{C}_{\infty}((t))$.

$\mathbb{G}_{a}:=$ the additive group.

$\mathrm{GL}_{r} / F:=$ for a field $F$, the $F$-group scheme of invertible $r \times r$ square matrices. $\mathbb{G}_{m}:=\mathrm{GL}_{1}$, the multiplicative group.

2.1.2. Block diagonal matrices. Let $A_{i} \in \operatorname{Mat}_{m_{i}}(\mathbb{L})$ for $i=1, \ldots, n$, and $m:=$ $m_{1}+\cdots+m_{n}$. We define $\bigoplus_{i=1}^{n} A_{i} \in \operatorname{Mat}_{m}(\mathbb{L})$ to be the canonical block diagonal matrix, that is, the matrix with $A_{1}, \ldots, A_{n}$ down the diagonal and zeros elsewhere.

2.1.3. $n$-fold twisting. For $n \in \mathbb{Z}$ and a formal Laurent series $f=\sum_{i} a_{i} t^{i} \in$ $\mathbb{C}_{\infty}((t))$, we define the $n$-fold twisting $f^{(n)}:=\sigma^{-n}(f):=\sum_{i} a_{i}^{p^{n}} t^{i}$. The $n$-fold twisting operation is an automorphism of the Laurent series field $\mathbb{C}_{\infty}((t))$ that stabilizes several subrings, for example, $\bar{k} \llbracket t \rrbracket, \bar{k}[t]$, and $\mathbb{T}$. More generally, for any matrix $B$ with entries in $\mathbb{C}_{\infty}((t))$, we define $B^{(n)}$ by the rule $B^{(n)}{ }_{i j}=B_{i j}{ }^{(n)}$. 
2.1.4. Entire power series. A power series $f=\sum_{i=0}^{\infty} a_{i} t^{i} \in \mathbb{C}_{\infty} \llbracket t \rrbracket$ that satisfies

$$
\lim _{i \rightarrow \infty} \sqrt[i]{\left|a_{i}\right|_{\infty}}=0
$$

and

$$
\left[k_{\infty}\left(a_{0}, a_{1}, a_{2}, \ldots\right): k_{\infty}\right]<\infty
$$

is called an entire power series. As a function of $t$, such a power series $f$ converges on all $\mathbb{C}_{\infty}$ and, when restricted to $\overline{k_{\infty}}, f$ takes values in $\overline{k_{\infty}}$. The ring of the entire power series is denoted by $\mathbb{E}$.

2.2. Pre-t-motives and the GP property. For $r$ a positive integer, let $\bar{k}(t)\left[\sigma^{r}, \sigma^{-r}\right]$ be the noncommutative ring of Laurent polynomials in $\sigma^{r}$ with coefficients in $\bar{k}(t)$, subject to the relation

$$
\sigma^{r} f:=f^{(-r)} \sigma^{r} \text { for all } f \in \bar{k}(t) .
$$

A pre- $t$-motive $M$ of level $r$ is a left $\bar{k}(t)\left[\sigma^{r}, \sigma^{-r}\right]$-module that is finite-dimensional over $\bar{k}(t)$. Letting $\mathbf{m} \in \operatorname{Mat}_{n \times 1}(M)$ comprise a $\bar{k}(t)$-basis of $M$, multiplication by $\sigma^{r}$ on $M$ is represented by

$$
\sigma^{r}(\mathbf{m})=\Phi \mathbf{m}
$$

for some matrix $\Phi \in \mathrm{GL}_{n}(\bar{k}(t))$. Furthermore, $M$ is called rigid analytically trivial if there exists $\Psi \in \mathrm{GL}_{n}(\mathbb{L})$ such that

$$
\sigma^{r}(\Psi):=\Psi^{(-r)}=\Phi \Psi .
$$

Such a matrix $\Psi$ is called a rigid analytic trivialization of the matrix $\Phi$. We also say that $\Psi$ is a rigid analytic trivialization of $M$ (with respect to $\mathbf{m}$ ). Note that if $\Psi^{\prime} \in \mathrm{GL}_{n}(\mathbb{L})$ is also a rigid analytic trivialization of $\Phi$, then by [Papanikolas 2008, $\S 4.1 .6]$ we have

$$
\Psi^{\prime-1} \Psi \in \mathrm{GL}_{n}\left(\mathbb{F}_{p^{r}}(t)\right) .
$$

Moreover, if we put $\mathbf{m}^{\prime}:=B \mathbf{m}$ for any fixed $B \in \mathrm{GL}_{n}(\bar{k}(t))$, then $\Phi^{\prime}:=B^{(-1)} \Phi B^{-1}$ represents multiplication by $\sigma^{r}$ on $M$ with respect to the $\bar{k}(t)$-basis $\mathbf{m}^{\prime}$ of $M$, and $\Psi^{\prime}:=B \Psi$ is a rigid analytic trivialization of $\Phi^{\prime}$.

Definition 2.2.1. Suppose we are given a rigid analytically trivial pre-t-motive $M$ of level $r$ that is of dimension $n$ over $\bar{k}(t)$. If there exists a $\bar{k}(t)$-basis $\mathbf{m} \in$ $\operatorname{Mat}_{n \times 1}(M)$ such that there exists $\Psi \in \mathrm{GL}_{n}(\mathbb{L}) \cap \operatorname{Mat}_{n}(\mathbb{E})$ that is a rigid analytic trivialization of $M$ with respect to $\mathbf{m}$ and satisfies

$$
\text { tr. } \operatorname{deg}_{\bar{k}(t)} \bar{k}(t)(\Psi)=\operatorname{tr} \cdot \operatorname{deg}_{\bar{k}} \bar{k}(\Psi(\theta)),
$$

then we say that $M$ has the GP property, where $\bar{k}(t)(\Psi)($ resp. $\bar{k}(\Psi(\theta)))$ is the field generated by all entries of $\Psi$ (resp. $\Psi(\theta)$ ) over $\bar{k}(t)$ (resp. $\bar{k}$ ). The GP property is independent of the choices of $\Psi$ for a fixed $\mathbf{m}$ because of (1). 
Given a rigid analytically trivial pre- $t$-motive $M$ of level $r$ with $(\mathbf{m}, \Phi, \Psi)$ as above, for any $s \in \mathbb{N}$ we define its $s$-th derived pre-t-motive $M^{(s)}$, which is a pre$t$-motive of level $r s$ : the underlying space of $M^{(s)}$ is the same as $M$, but it is now regarded as a left $\bar{k}(t)\left[\sigma^{r s}, \sigma^{-r s}\right]$-module. Letting

$$
\Phi^{\prime}:=\Phi^{(-(s-1) r)} \ldots \Phi^{(-r)} \Phi,
$$

we have $\sigma^{r s} \mathbf{m}=\Phi^{\prime} \mathbf{m}$ and $\sigma^{r s} \Psi:=\Psi^{(-r s)}=\Phi^{\prime} \Psi$, and hence $\Psi$ is also a rigid analytic trivialization of $M^{(s)}$.

Proposition 2.2.2. Let $M$ be a rigid analytically trivial pre-t-motive of level $r$ that has the GP property. For any positive integer $s$, the $s$-th derived pre-t-motive $M^{(s)}$ of $M$ is also rigid analytically trivial and has the GP property.

Theorem 2.2.3 [Chang 2009, Theorem 1.2; Papanikolas 2008, Theorem 5.2.2]. Suppose $\Phi \in \operatorname{Mat}_{n}(\bar{k}[t])$ defines a rigid analytically trivial pre-t-motive $M$ of level $r$ with a rigid analytic trivialization $\Psi \in \operatorname{Mat}_{n}(\mathbb{T}) \cap \mathrm{GL}_{n}(\mathbb{L})$. If $\operatorname{det} \Phi(0) \neq 0$ and $\operatorname{det} \Phi\left(\theta^{1 / p^{r i}}\right) \neq 0$ for all $i=1,2,3, \ldots$, then $M$ has the GP property.

By [Anderson et al. 2004, Proposition 3.1.3], the condition det $\Phi(0) \neq 0$ implies $\Psi \in \mathrm{Mat}_{n}(\mathbb{E})$. Combining Theorem 2.2.3 and Proposition 2.2.2, we have:

Corollary 2.2.4. Given an integer $d \geq 2$, we let $\ell:=\operatorname{lcm}(1, \ldots, d)$. For each $1 \leq r \leq d$, let $\ell_{r}:=\ell / r$ and let $\Phi_{r} \in \operatorname{Mat}_{n_{r}}(\bar{k}[t]) \cap \mathrm{GL}_{n_{r}}(\bar{k}(t))$ define a pre-tmotive $M_{r}$ of level $r$ with a rigid analytic trivialization $\Psi_{r} \in \operatorname{Mat}_{n_{r}}(\mathbb{T}) \cap \mathrm{GL}_{n_{r}}(\mathbb{L})$. Suppose that each $\Phi_{r}$ satisfies the hypotheses of Theorem 2.2 .3 for $r=1, \ldots, d$. Then the direct sum

$$
M:=\bigoplus_{r=1}^{d} M_{r}{ }^{\left(\ell_{r}\right)}
$$

is a rigid analytically trivial pre-t-motive of level $\ell$ that has the GP property.

Proof. For each $1 \leq r \leq d$, we define

$$
\Phi_{r}^{\prime}:=\Phi_{r}^{\left(-\left(\ell_{r}-1\right) r\right)} \cdots \Phi_{r}^{(-r)} \Phi_{r} .
$$

Moreover, if we define

$$
\Phi^{\prime}:=\oplus_{r=1}^{d} \Phi_{r}^{\prime}, \quad \Psi^{\prime}:=\oplus_{r=1}^{d} \Psi_{r},
$$

then we have

$$
\Psi^{\prime(-\ell)}=\Phi^{\prime} \Psi^{\prime} .
$$

Note that the matrix representing multiplication by $\sigma^{\ell}$ on $M$ with respect to the evident $\bar{k}(t)$-basis is given by $\Phi^{\prime}$.

Our task is to show that $\Phi^{\prime}$ satisfies the hypotheses of Theorem 2.2.3 (with respect to the operator $\sigma^{\ell}$ ), whence the result. It is obvious that $\operatorname{det} \Phi^{\prime}(0) \neq 0$ 
since $\operatorname{det} \Phi_{r}(0) \neq 0$ for each $1 \leq r \leq d$. Suppose that $\operatorname{det} \Phi^{\prime}\left(\theta^{1 / p^{\ell j}}\right)=0$ for some $j \in \mathbb{N}$. This implies that there exists $1 \leq r \leq d$ and $0 \leq m \leq \ell_{r}-1$ such that

$$
\operatorname{det} \Phi_{r}^{(-r m)}\left(\theta^{(-\ell j)}\right)=0
$$

However, this is equivalent to

$$
\operatorname{det} \Phi_{r}\left(\theta^{(-(\ell j-r m))}\right)=0 .
$$

Since $0 \leq m \leq \ell_{r}-1$ and $r \mid \ell$, we have that $(\ell j-r m)>0$ and $r \mid(\ell j-r m)$. Thus, (2) contradicts the hypothesis that $\operatorname{det} \Phi_{r}\left(\theta^{(-r h)}\right) \neq 0$ for all $h=1,2,3, \ldots$

2.3. Difference Galois groups and transcendence. In this section, we review the related theory developed in [Papanikolas 2008]. Let $r$ be a fixed positive integer. The category of pre-t-motives of level $r$ forms a rigid abelian $\mathbb{F}_{p^{r}}(t)$-linear tensor category. Also, the category $\mathscr{R}$ of rigid analytically trivial pre-t-motives of level $r$ forms a neutral Tannakian category over $\mathbb{F}_{p^{r}}(t)$. Given an object $M$ in $\mathscr{R}$, we let $\mathscr{R}_{M}$ be the strictly full Tannakian subcategory of $\mathscr{R}$ generated by $M$. That is, $\mathscr{R}_{M}$ consists of all objects of $\mathscr{R}$ isomorphic to subquotients of finite direct sums of

$$
M^{\otimes u} \otimes\left(M^{\vee}\right)^{\otimes v} \text { for various } u, v,
$$

where $M^{\vee}$ is the dual of $M$. By Tannakian duality, $\mathscr{R}_{M}$ is representable by an affine algebraic group scheme $\Gamma_{M}$ over $\mathbb{F}_{p^{r}}(t)$. The group $\Gamma_{M}$ is called the Galois group of $M$ and it is described explicitly as follows.

Suppose that $\Phi \in \mathrm{GL}_{n}(\bar{k}(t))$ provides multiplication by $\sigma^{r}$ on $M$ with respect to a fixed basis $\mathbf{m} \in \operatorname{Mat}_{n \times 1}(M)$ over $\bar{k}(t)$. Let $\Psi \in \mathrm{GL}_{n}(\mathbb{L})$ be a rigid analytic trivialization for $\Phi$. Let $X:=\left(X_{i j}\right)$ be an $n \times n$ matrix whose entries are independent variables $X_{i j}$, and define a $\bar{k}(t)$-algebra homomorphism $v: \bar{k}(t)[X, 1 / \operatorname{det} X] \rightarrow \mathbb{L}$ such that $v\left(X_{i j}\right)=\Psi_{i j}$ for all $1 \leq i, j \leq n$. We let

$$
\begin{aligned}
& \Sigma_{\Psi}:=\operatorname{im} v=\bar{k}(t)[\Psi, 1 / \operatorname{det} \Psi] \subseteq \mathbb{L}, \\
& Z_{\Psi}:=\operatorname{Spec} \Sigma_{\Psi} .
\end{aligned}
$$

Then $Z_{\Psi}$ is a closed $\bar{k}(t)$-subscheme of $\mathrm{GL}_{n} / \bar{k}(t)$. Let $\Psi_{1}, \Psi_{2} \in \mathrm{GL}_{n}\left(\mathbb{L} \otimes_{\bar{k}(t)} \mathbb{\mathbb { L }}\right)$ be the matrices satisfying $\left(\Psi_{1}\right)_{i j}=\Psi_{i j} \otimes 1$ and $\left(\Psi_{2}\right)_{i j}=1 \otimes \Psi_{i j}$ for all $1 \leq i, j \leq n$. Let $\widetilde{\Psi}:=\Psi_{1}^{-1} \Psi_{2}$. We have an $\mathbb{F}_{p^{r}}(t)$-algebra homomorphism $\mu: \mathbb{F}_{p^{r}}(t)[X, 1 / \operatorname{det} X] \rightarrow$ $\mathbb{L} \otimes_{\bar{k}(t)} \mathbb{L}$ such that $\mu\left(X_{i j}\right)=\widetilde{\Psi}_{i j}$ for all $1 \leq i, j \leq n$. Furthermore, we define

$$
\begin{aligned}
\Delta & :=\operatorname{im} \mu, \\
\Gamma_{\Psi} & :=\operatorname{Spec} \Delta .
\end{aligned}
$$


Theorem 2.3.1 [Papanikolas 2008, Theorems 4.2.11, 4.3.1, 4.5.10]. The scheme $\Gamma_{\Psi}$ is a closed $\mathbb{F}_{p^{r}}(t)$-subgroup scheme of $\mathrm{GL}_{n} / \mathbb{F}_{p^{r}}(t)$, which is isomorphic to the Galois group $\Gamma_{M}$ over $\mathbb{F}_{p^{r}}(t)$. Moreover, $\Gamma_{\Psi}$ has the following properties:

(a) $\Gamma_{\Psi}$ is smooth over $\overline{\mathbb{F}_{p}(t)}$ and is geometrically connected.

(b) $\operatorname{dim} \Gamma_{\Psi}=\operatorname{tr} \cdot \operatorname{deg}_{\bar{k}(t)} \bar{k}(t)(\Psi)$.

(c) $Z_{\Psi}$ is a $\Gamma_{\Psi}$-torsor over $\bar{k}(t)$.

In particular, if $M$ has the GP property, then

(d) $\operatorname{dim} \Gamma_{\Psi}=\operatorname{tr} \cdot \operatorname{deg}_{\bar{k}} \bar{k}(\Psi(\theta))$.

We call $\Gamma_{\Psi}$ the Galois group associated to the difference equation $\Psi^{(-r)}=\Phi \Psi$. This $\Gamma_{\Psi}$ is independent of the analytic trivialization $\Psi$, up to isomorphism over $\mathbb{F}_{p^{r}}(t)$. Throughout this paper we always identify $\Gamma_{M}$ with $\Gamma_{\Psi}$, and regard it as a linear algebraic group over $\mathbb{F}_{p^{r}}(t)$ because of Theorem 2.3.1(a).

Remark 2.3.2. Let $n_{1}, n_{2}$ be positive integers and $\mathbf{0}:=0_{n_{1} \times n_{2}}$ be the zero matrix of size $n_{1} \times n_{2}$. Suppose that the matrix

$$
\Phi:=\left[\begin{array}{cc}
\Phi_{1} & \mathbf{0} \\
\Phi_{3} & \Phi_{2}
\end{array}\right] \in \mathrm{GL}_{n_{1}+n_{2}}(\bar{k}(t))
$$

defines a rigid analytically trivial pre- $t$-motive $M$ of level $r$. Then one can always find its rigid analytic trivialization of the form

$$
\Psi:=\left[\begin{array}{cc}
\Psi_{1} & 0 \\
\Psi_{3} & \Psi_{2}
\end{array}\right] \in \mathrm{GL}_{n_{1}+n_{2}}(\mathbb{L})
$$

By (3), we have that

$$
\Gamma_{\Psi} \subseteq\left\{\left[\begin{array}{cc}
* \mathbf{0} \\
* *
\end{array}\right]\right\} \subseteq \mathrm{GL}_{n_{1}+n_{2}} / \mathbb{F}_{p^{r}}(t)
$$

Let $N$ be the sub-pre-t-motive (of level $r$ ) of $M$ defined by $\Phi_{1} \in \mathrm{GL}_{n_{1}}(\bar{k}(t)$ ) with rigid analytic trivialization $\Psi_{1}$; then by the Tannakian theory we have a natural surjective morphism

$$
\begin{aligned}
\pi: \Gamma_{\Psi}\left(\overline{\mathbb{F}_{p}(t)}\right) & \rightarrow \Gamma_{\Psi_{1}}\left(\overline{\mathbb{F}_{p}(t)}\right), \\
\gamma & \mapsto \pi(\gamma) .
\end{aligned}
$$

In fact, $\pi(\gamma)$ comes from the restriction of the action of $\gamma$ to the fiber functor of $\mathscr{R}_{N}$ (which is a full subcategory of $\mathscr{R}_{M}$ ). Precisely, $\pi(\gamma)$ is the matrix cut out from the upper left square of $\gamma$ with size $n_{1}$ (for detailed arguments, see [Papanikolas 2008, §6.2.2]). 


\section{A dimension criterion}

3.1. Pre-t-motives of type SV. Let $r$ be a fixed positive integer and let $\left\{n_{1}, \ldots, n_{h}\right\}$ be $h$ nonnegative integers. We say that a pre-t-motive $M$ of level $r$ is of type SV (its Galois group being an extension of a split torus by a vector group) if the multiplication by $\sigma^{r}$ on $M$ is represented by the matrix

$$
\Phi:=\bigoplus_{i=1}^{h} A_{i}, \quad A_{i}:=\left[\begin{array}{cccc}
a_{i} & 0 & \cdots & 0 \\
a_{i 1} & 1 & \cdots & 0 \\
\vdots & \vdots & \ddots & \vdots \\
a_{i n_{i}} & 0 & \cdots & 1
\end{array}\right] \in \mathrm{GL}_{1+n_{i}}(\bar{k}(t))
$$

which has rigid analytic trivialization

$$
\Psi:=\bigoplus_{i=1}^{h} F_{i}, \quad F_{i}:=\left[\begin{array}{cccc}
f_{i} & 0 & \cdots & 0 \\
f_{i 1} & 1 & \cdots & 0 \\
\vdots & \vdots & \ddots & \vdots \\
f_{i n_{i}} & 0 & \cdots & 1
\end{array}\right] \in \mathrm{GL}_{1+n_{i}}(\mathbb{L}) .
$$

Let $T$ be the Galois group associated to the difference equation

$$
\left[\begin{array}{ccc}
f_{1} & \cdots & 0 \\
\vdots & \ddots & \vdots \\
0 & \cdots & f_{h}
\end{array}\right]^{(-r)}=\left[\begin{array}{ccc}
a_{1} & \cdots & 0 \\
\vdots & \ddots & \vdots \\
0 & \cdots & a_{h}
\end{array}\right]\left[\begin{array}{ccc}
f_{1} & \cdots & 0 \\
\vdots & \ddots & \vdots \\
0 & \cdots & f_{h}
\end{array}\right],
$$

and note that by (3), $T$ is a subtorus of the $h$-dimensional split torus in $\mathrm{GL}_{h} / \mathbb{F}_{p^{r}}(t)$. By the same reason as (4), we have the natural projection of Galois groups

$$
\Gamma_{\Psi} \rightarrow T
$$

given in terms of coordinates by

$$
\bigoplus_{i=1}^{h}\left[\begin{array}{cccc}
x_{i} & 0 & \cdots & 0 \\
x_{i 1} & 1 & \cdots & 0 \\
\vdots & \vdots & \ddots & \vdots \\
x_{i n_{i}} & 0 & \cdots & 1
\end{array}\right] \mapsto\left[x_{1}\right] \oplus \cdots \oplus\left[x_{h}\right]
$$

One has also the exact sequence of linear algebraic groups

$$
1 \rightarrow V \rightarrow \Gamma_{\Psi} \rightarrow T \rightarrow 1
$$


where $V$ is a vector group contained in the $\left(\sum_{i=1}^{h} n_{i}\right)$-dimensional "coordinate" vector group $G$, which is defined as the set of matrices

$$
\bigoplus_{i=1}^{h}\left[\begin{array}{cccc}
1 & 0 & \cdots & 0 \\
x_{i 1} & 1 & \cdots & 0 \\
\vdots & \vdots & \ddots & \vdots \\
x_{i n_{i}} & 0 & \cdots & 1
\end{array}\right]
$$

with usual multiplication. This subgroup $V$ is the unipotent radical of the (solvable) Galois group $\Gamma_{\Psi}$, and $\operatorname{dim} \Gamma_{\Psi}=\operatorname{dim} V+\operatorname{dim} T$.

Definition 3.1.1. Let $M$ be a rigid analytically trivial pre- $t$-motive of level $r$ that is of $\mathbf{S V}$ type given as above. We say that its Galois group $\Gamma_{\Psi}$ is full if $\operatorname{dim} V=$ $\sum_{i=1}^{h} n_{i}$, that is, $V=G$.

3.2. Criterion for direct sum motives to have full Galois group. We continue with the notation of Section 3.1. For each $i, 1 \leq i \leq h$, the $1 \times 1$ matrix $\left[a_{i}\right]$ defines a sub-pre-t-motive (of level $r$ ) of $M$, which is one-dimensional over $\bar{k}(t)$. Its rigid analytic trivialization is given by $f_{i}$ satisfying

$$
\left[f_{i}\right]^{(-r)}=\left[a_{i}\right]\left[f_{i}\right] .
$$

We call one such sub-pre-t-motive of level $r$ a diagonal of the pre-t-motive $M$. By Theorem 2.3.1, the Galois group of a diagonal of $M$ is $\mathbb{G}_{m}$ if and only if the corresponding trivialization $f_{i}$ is transcendental over $\bar{k}(t)$. In this situation, the canonical projection $T \rightarrow \mathbb{G}_{m}$ on the $i$-th coordinate of $T$ is surjective.

There is a canonical action of $T$ on $G$ given in terms of coordinates by

$$
\left(\left[x_{1}\right] \oplus \cdots \oplus\left[x_{h}\right]\right) *\left(\oplus_{i=1}^{h}\left[\begin{array}{cccc}
1 & 0 & \cdots & 0 \\
x_{i 1} & 1 & \cdots & 0 \\
\vdots & \vdots & \ddots & \vdots \\
x_{i n_{i}} & 0 & \cdots & 1
\end{array}\right]\right):=\bigoplus_{i=1}^{h}\left[\begin{array}{cccc}
1 & 0 & \cdots & 0 \\
x_{i} x_{i 1} & 1 & \cdots & 0 \\
\vdots & \vdots & \ddots & \vdots \\
x_{i} x_{i n_{i}} & 0 & \cdots & 1
\end{array}\right] .
$$

This action induces an action of $T$ on $V$ compatible with the one coming from (6). Hence, we have that given any element $\gamma \in V$ whose $x_{i j}$-coordinate is nonzero, the orbit inside $V$ given by the action of $T$ on $\gamma$ must be infinite if $f_{i}$ is transcendental over $\bar{k}(t)$.

Definition 3.2.1. Let $M_{i}$ be a rigid analytically trivial pre- $t$-motive of level $r$ that is of type $\mathbf{S V}$ for $i=1, \ldots, d$. We say that this set of pre- $t$-motives $\left\{M_{i}\right\}_{i=1}^{d}$ is diagonally independent if for any $1 \leq i, j \leq d, i \neq j$, the Galois group of $N_{i} \oplus N_{j}$ is a two-dimensional torus over $\mathbb{F}_{p^{r}}(t)$, where $N_{i}$ is any diagonal of $M_{i}$ and $N_{j}$ is any diagonal of $M_{j}$. 
Theorem 3.2.2. Given any positive integer $r$, let $M_{1}, \ldots, M_{d}$ be rigid analytically trivial pre-t-motives of level $r$ that are of type $\mathbf{S V}$ and have full Galois groups. Suppose that the set $\left\{M_{i}\right\}_{i=1}^{d}$ is diagonally independent. Then the Galois group of $M:=\bigoplus_{i=1}^{d} M_{i}$ is also full.

Proof. We first explain that without loss of generality, we may assume $d=2$. We prove the result by induction on $d$. Consider $M=\left(\bigoplus_{i=1}^{d-1} M_{i}\right) \oplus M_{d}$. Then the induction hypothesis implies that the Galois group of $\left(\bigoplus_{i=1}^{d-1} M_{i}\right)$ is full. Since the following argument of the case $d=2$ can be applied to the computation of the Galois group of the direct sum of $\left(\bigoplus_{i=1}^{d-1} M_{i}\right)$ and $M_{d}$, we may assume $d=2$.

Now we set $d=2$ and let the pre- $t$-motive $M_{i}$ be defined by a matrix $\Phi_{i}$, for $i=1,2$, with $\Psi=\Psi_{1} \oplus \Psi_{2}$ a rigid analytic trivialization of $M$, and let $\Gamma_{\Psi}, \Gamma_{\Psi_{1}}$, $\Gamma_{\Psi_{2}}$ be the Galois groups. The unipotent radicals of these groups are denoted by $V, V_{1}, V_{2}$ respectively. Let $G, G_{1}, G_{2}$ be the coordinate vector groups containing $V, V_{1}, V_{2}$, respectively. Suppose the matrix $\Phi_{1}$ has $h$ diagonal blocks, and let the coordinates of $G_{1}$ be denoted by $x_{i j}, i=1, \ldots, h, j=1, \ldots, n_{i}$. Similarly, let $y_{i j}$, $i=1, \ldots, \ell, j=1, \ldots, m_{i}$ denote the coordinates of $G_{2}$. Any subspace $W \subseteq G$ obtained by setting some of these coordinates to 0 is called a linear coordinate subspace. The hypothesis that the Galois group of $M_{i}$ is full means exactly that $G_{i}=V_{i}$, for $i=1,2$. We are going to prove that $G=V$.

Suppose $V$ has codimension $s$ in the coordinate vector group $G$. We can find a linear coordinate subspace $W \subseteq G$ of dimension $s$ such that $W \cap V$ is of dimension 0 . Since $W \cap V$ is invariant under $T$, it must be equal to the neutral element of $G$, because the hypothesis that $M_{1}$ and $M_{2}$ are diagonally independent implies in particular that the Galois group of any diagonal of $M$ is $\mathbb{G}_{m}$.

Let $W^{\prime} \subseteq G$ be the linear coordinate space given by those coordinates disjoint from those of $W$. Then the natural projection from $G$ to $W^{\prime}$ induces on $V$ an isomorphism of vector groups. Composing the inverse of this isomorphism with the surjective morphism $\pi_{1}$ in the diagram

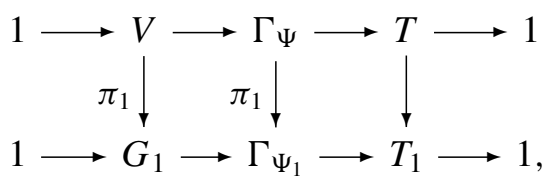

we obtain a morphism $\pi_{1}$ from $W^{\prime}$ onto $G_{1}$ that is furthermore a $T$-morphism.

We contend that under the hypothesis that $M_{1}$ and $M_{2}$ are diagonally independent, $\pi_{1}$ maps $G_{2} \cap W^{\prime}$ to zero. This contention results from the following basic lemma by taking any diagonal $N_{1}\left(\right.$ resp. $N_{2}$ ) of $M_{1}$ (resp. $M_{2}$ ) and considering the restriction of the above morphism $\pi_{1}$ to a single block. Now since $G=G_{1} \times G_{2}$, it follows that $\pi_{1}\left(G_{1} \cap W^{\prime}\right)=G_{1}$. Thus $G_{1} \subseteq W^{\prime}$. Similarly we also have $G_{2} \subseteq W^{\prime}$, and hence $G=W^{\prime}$ and $G=V$. 
Lemma 3.2.3. Let $G_{1}$ (resp. $G_{2}$ ) be the vector group with coordinates

$$
\left[\begin{array}{cccc}
1 & 0 & \cdots & 0 \\
x_{1} & 1 & \cdots & 0 \\
\vdots & \vdots & \ddots & \vdots \\
x_{n} & 0 & \cdots & 1
\end{array}\right], \quad\left(\text { resp. }\left[\begin{array}{cccc}
1 & 0 & \cdots & 0 \\
y_{1} & 1 & \cdots & 0 \\
\vdots & \vdots & \ddots & \vdots \\
y_{m} & 0 & \cdots & 1
\end{array}\right]\right)
$$

Let $\mathbb{G}_{m}^{2}$ act on $G_{1}\left(\right.$ resp. $\left.G_{2}\right)$ by

$$
\mathbb{G}_{m}^{2} \ni(x, y): x_{i} \mapsto x x_{i}, 1 \leq i \leq n, \quad\left(\text { resp. } y_{j} \mapsto y y_{j}, 1 \leq j \leq m\right) .
$$

If $\pi_{1}: G_{2} \rightarrow G_{1}$ is a $\mathbb{G}_{m}^{2}$-morphism, then $\pi_{1} \equiv 0$.

\section{Application to zeta values}

Throughout this section, we use $r i$ for two subscripts (for example, $D_{r i}$ depends on the two parameters $r$ and $i$ ), and use $*, r n$ for three subscripts (for example, $L_{\alpha, r n}$ depends on the three parameters $\alpha, r$ and $n$ ).

4.1. Carlitz theory. Throughout Section 4 we fix a positive integer $r$. Recall the Carlitz $\mathbb{F}_{p^{r}}[t]$-module, denoted by $C_{r}$, which is given by the $\mathbb{F}_{p^{r}}$-linear ring homomorphism

$$
C_{r}=\left(t \mapsto\left(x \mapsto \theta x+x^{p^{r}}\right)\right): \mathbb{F}_{p^{r}}[t] \rightarrow \operatorname{End}_{\mathbb{F}_{p^{r}}}\left(\mathbb{G}_{a}\right) .
$$

Note that when we regard $C_{r}$ as a Drinfeld $\mathbb{F}_{p}[t]$-module, it is of rank $r$ [Goss 1996; Thakur 2004]. One has the Carlitz exponential associated to $C_{r}$ :

$$
\exp _{C_{r}}(z):=\sum_{i=0}^{\infty} \frac{z^{p^{r i}}}{D_{r i}}
$$

Here we set

$$
D_{r 0}:=1, \quad D_{r i}:=\prod_{j=0}^{i-1}\left(\theta^{p^{r i}}-\theta^{p^{r j}}\right), \quad i \geq 1 .
$$

Now $\exp _{C_{r}}(z)$ is an entire power series in $z$ satisfying the functional equation

$$
\exp _{C_{r}}(\theta z)=\theta \exp _{C_{r}}(z)+\exp _{C_{r}}(z)^{p^{r}} .
$$

Moreover one has the product expansion

$$
\exp _{C_{r}}(z)=z \prod_{0 \neq a \in \mathbb{F}_{p^{r}}[\theta]}\left(1-\frac{z}{a \tilde{\pi}_{r}}\right)
$$

where

$$
\tilde{\pi}_{r}=\theta(-\theta)^{1 /\left(p^{r}-1\right)} \prod_{i=1}^{\infty}\left(1-\theta^{1-p^{r i}}\right)^{-1}
$$


is a fundamental period of $C_{r}$. We fix once and for all a choice of $(-\theta)^{1 /\left(p^{r}-1\right)}$ so that $\tilde{\pi}_{r}$ is a well-defined element in $\overline{\mathbb{F}_{p}((1 / \theta))}$. We also choose these roots in a compatible way so that when $r$ divides $r^{\prime}$, the number $(-\theta)^{1 /\left(p^{r}-1\right)}$ is a power of $(-\theta)^{1 /\left(p^{r^{\prime}}-1\right)}$.

The formal inverse of Carlitz exponential is the Carlitz logarithm. It is the power series

$$
\log _{C_{r}}(z)=\sum_{i=0}^{\infty} \frac{z^{p^{r i}}}{L_{r i}}
$$

where

$$
L_{r 0}:=1, \quad L_{r i}:=\prod_{j=1}^{i}\left(\theta-\theta^{p^{r j}}\right) .
$$

As a function in $z, \log _{C_{r}}(z)$ converges for all $z \in \mathbb{C}_{\infty}$ with $|z|_{\infty}<|\theta|_{\infty}^{p^{r} /\left(p^{r}-1\right)}$. It satisfies the functional equation

$$
\theta \log _{C_{r}}(z)=\log _{C_{r}}(\theta z)+\log _{C_{r}}\left(z^{p^{r}}\right)
$$

whenever the values in question are defined.

For a positive integer $n$, the $n$-th Carlitz polylogarithm associated to $C_{r}$ is the series

$$
\operatorname{Plog}_{r n}(z):=\sum_{i=0}^{\infty} \frac{z^{p^{r i}}}{L_{r i}^{n}}
$$

which converges $\infty$-adically for all $z \in \mathbb{C}_{\infty}$ with $|z|_{\infty}<|\theta|_{\infty}^{n p^{r} /\left(p^{r}-1\right)}$. Its value at a particular $z=\alpha \neq 0$ is called the $n$-th polylogarithm of $\alpha$ associated to $C_{r}$. In transcendence theory we are interested in those polylogarithms of $\alpha \in \bar{k}^{\times}$, as analogous to classical logarithms of algebraic numbers.

4.2. Algebraic independence of special functions. For any positive integer $r$, let

$$
\Omega_{r}(t):=(-\theta)^{-p^{r} /\left(p^{r}-1\right)} \prod_{i=1}^{\infty}\left(1-\frac{t}{\theta^{p^{r i}}}\right) \in \overline{k_{\infty}} \llbracket t \rrbracket \subseteq \mathbb{C}_{\infty}((t)) .
$$

$\Omega_{r} \in \mathbb{E}$, since $\left|\theta^{p^{r i}}\right|_{\infty} \rightarrow \infty$. Furthermore, $\Omega_{r}$ satisfies the functional equation

$$
\Omega_{r}^{(-r)}(t)=(t-\theta) \Omega_{r}(t),
$$

and its specialization at $t=\theta$ gives $\Omega_{r}(\theta)=-1 / \tilde{\pi}_{r}$.

By (8), the function $\Omega_{r}$ provides a rigid analytic trivialization of the Carlitz motive $\mathscr{C}_{r}$ of level $r$ that has the GP property (see Theorem 2.2.3). This is the pre- $t$-motive with underlying space $\bar{k}(t)$ itself and $\sigma^{r}$ acts by $\sigma^{r} f=(t-\theta) f^{(-r)}$ for $f \in \mathscr{C}_{r}$.

For any $d \in \mathbb{N}$, we let $\ell:=\operatorname{lcm}(1, \ldots, d)$ and $\ell_{r}:=\ell / r$ for $r=1, \ldots, d$. We let $\mathscr{C}_{r}^{\left(\ell_{r}\right)}$ be the $\ell_{r}$-th derived pre-t-motive of $\mathscr{C}_{r}$ that is a rigid analytically 
trivial pre-t-motive of level $\ell$ (see Section 2.2). Then we define the direct sum $M=M_{d}:=\bigoplus_{r=1}^{d} \mathscr{b}_{r}^{\left(\ell_{r}\right)}$. By Corollary 2.2.4, $M$ also has the GP property. We note that the canonical rigid analytical trivialization of $M$ is the diagonal matrix $\Psi \in \operatorname{Mat}_{d}(\mathbb{E}) \cap \mathrm{GL}_{d}(\mathbb{L})$ with diagonal entries $\Omega_{1}, \ldots, \Omega_{d}$.

Lemma 4.2.1. Given any positive integer $d \geq 2$, we let $\ell:=\operatorname{lcm}(1, \ldots, d)$. Let $M=M_{d}$ be the rigid analytically trivial pre-t-motive of level $\ell$ with rigid analytic trivialization $\Psi$ defined as above. Then we have $\operatorname{dim} \Gamma_{\Psi}=d$. In particular, the functions $\Omega_{1}, \ldots, \Omega_{d}$ are algebraically independent over $\vec{k}(t)$ and the values $\tilde{\pi}_{1}, \ldots, \tilde{\pi}_{d}$ are algebraically independent over $\bar{k}$.

Proof. Suppose $\operatorname{dim} \Gamma_{\Psi}<d$. Since $\Psi$ is a diagonal matrix with diagonal entries $\Omega_{1}, \ldots, \Omega_{d}$, by (3) we have that $\Gamma_{\Psi} \subseteq \mathrm{T}$, where $\mathrm{T}$ is the split torus of dimension $d$ in $\mathrm{GL}_{d} / \mathbb{F}_{p^{\ell}}(t)$. We let $X_{1}, \ldots, X_{d}$ be the coordinates of $\mathrm{T}$ and $\chi_{j}$ the character of $\mathrm{T}$ that projects the $j$-th diagonal position to $\mathbb{G}_{m}$. Note that $\left\{\chi_{j}\right\}_{j=1}^{d}$ generates the character group of T. Hence $\Gamma_{\Psi}$ is the kernel of some characters of $\mathrm{T}$, that is, canonical generators of the defining ideal for $\Gamma_{\Psi}$ can be of the form $X_{1}^{m_{1}} \cdots X_{d}^{m_{d}}-1$ for some integers $m_{1}, \ldots, m_{d}$, not all zero. By (3) we have that

$$
\left(\Omega_{1}^{-m_{1}} \ldots \Omega_{d}^{-m_{d}}\right) \otimes\left(\Omega_{1}^{m_{1}} \ldots \Omega_{d}^{m_{d}}\right)=1 \in \mathbb{L} \otimes_{\bar{k}(t)} \mathbb{L},
$$

and hence

$$
\beta:=\Omega_{1}^{m_{1}} \ldots \Omega_{d}^{m_{d}} \in \bar{k}(t)^{\times} .
$$

We recall that $\Omega_{r}$ has zeros on $\left\{\theta^{p^{r j}}\right\}_{j=1}^{\infty}$. Since $\beta \in \bar{k}(t)^{\times}$, it has only finitely many zeros and poles, and hence ord ${ }_{t=\theta^{p^{h}}}(\beta)=0$ for $h \gg 0$. Choose a prime number $p^{\prime}$ sufficiently large that

- $p^{\prime}>d$, and

- the order of vanishing of $\beta$ at $t=\theta^{p^{p^{\prime}}}$ is zero.

These conditions imply that $m_{1}=0$ from (9). Iterating this argument, we conclude that $m_{1}=\cdots=m_{d}=0$, a contradiction.

4.3. Algebraic independence of polylogarithms. Given $n \in \mathbb{N}$ and $\alpha \in \bar{k}^{\times}$with $|\alpha|_{\infty}<|\theta|_{\infty}^{n p^{r} /\left(p^{r}-1\right)}$, we consider the power series

$$
L_{\alpha, r n}(t):=\alpha+\sum_{i=1}^{\infty} \frac{\alpha^{p^{r i}}}{\left(t-\theta^{p^{r}}\right)^{n} \cdots\left(t-\theta^{p^{r i}}\right)^{n}},
$$

which as a function on $\mathbb{C}_{\infty}$ converges on $|t|_{\infty}<|\theta|_{\infty}^{p^{r}}$. We note that $L_{\alpha, r n}(\theta)$ is exactly the $n$-th polylogarithm of $\alpha$ associated to $C_{r}$, that is,

$$
L_{\alpha, r n}(\theta)=\operatorname{Plog}_{r n}(\alpha) .
$$


Given a collection of such numbers $\alpha$, say $\alpha_{1}, \ldots, \alpha_{m}$, we define

$$
\Phi_{r n}\left(\alpha_{1}, \ldots, \alpha_{m}\right):=\left[\begin{array}{cccc}
(t-\theta)^{n} & 0 & \cdots & 0 \\
\alpha_{1}^{(-r)}(t-\theta)^{n} & 1 & \cdots & 0 \\
\vdots & \vdots & \ddots & \vdots \\
\alpha_{m}^{(-r)}(t-\theta)^{n} & 0 & \cdots & 1
\end{array}\right] \in \operatorname{GL}_{m+1}(\bar{k}(t)) \cap \operatorname{Mat}_{m+1}(\bar{k}[t])
$$

and

$$
\Psi_{r n}\left(\alpha_{1}, \ldots, \alpha_{m}\right):=\left[\begin{array}{cccc}
\Omega_{r}^{n} & 0 & \cdots & 0 \\
\Omega_{r}^{n} L_{\alpha_{1}, r n} & 1 & \cdots & 0 \\
\vdots & \vdots & \ddots & \vdots \\
\Omega_{r}^{n} L_{\alpha_{m}, r n} & 0 & \cdots & 1
\end{array}\right] \in \mathrm{GL}_{m+1}(\mathbb{L}) \cap \operatorname{Mat}_{m+1}(\mathbb{E}) .
$$

Then one has [Chang and Yu 2007, §3.1.2]

$$
\Psi_{r n}\left(\alpha_{1}, \ldots, \alpha_{m}\right)^{(-r)}=\Phi_{r n}\left(\alpha_{1}, \ldots, \alpha_{m}\right) \Psi_{r n}\left(\alpha_{1}, \ldots, \alpha_{m}\right) .
$$

Hence, $\Phi_{r n}\left(\alpha_{1}, \ldots, \alpha_{m}\right)$ defines a rigid analytically trivial pre-t-motive of level $r$ that has the GP property.

In [Chang and Yu 2007], we followed Papanikolas' methods to generalize the algebraic independence of Carlitz logarithms to algebraic independence of polylogarithms. Precisely, by [Chang and Yu 2007, Theorem 3.1] and Theorem 2.2.3 we have:

Theorem 4.3.1. Given any positive integers $r$ and $n$, let $\alpha_{1}, \ldots, \alpha_{m} \in \bar{k}^{\times}$satisfy $\left|\alpha_{i}\right|_{\infty}<|\theta|_{\infty}^{n p^{r} /\left(p^{r}-1\right)}$ for $i=1, \ldots, m$. Then

$$
\begin{aligned}
\operatorname{dim}_{\mathbb{F}_{p^{r}}(\theta)} N_{r n} & =\operatorname{tr} \cdot \operatorname{deg}_{\bar{k}} \bar{k}\left(\tilde{\pi}_{r}^{n}, L_{\alpha_{1}, r n}(\theta), \ldots, L_{\alpha_{m}, r n}(\theta)\right) \\
& =\operatorname{tr} \cdot \operatorname{deg}_{\bar{k}(t)} \bar{k}(t)\left(\Omega_{r}^{n}, L_{\alpha_{1}, r n}, \ldots, L_{\alpha_{m}, r n}\right),
\end{aligned}
$$

where

$$
N_{r n}:=\mathbb{F}_{p^{r}}(\theta)-\operatorname{Span}\left\{\tilde{\pi}_{r}^{n}, L_{\alpha_{1}, r n}(\theta), \ldots, L_{\alpha_{m}, r n}(\theta)\right\} .
$$

\subsection{Formulas for zeta values.}

4.4.1. Euler-Carlitz relations. Fix a positive integer $r$ for this subsection. Carlitz [1935] introduced the power sum

$$
\left.\zeta_{r}(n):=\sum_{\substack{a \in \mathbb{F}_{p^{r}}[\theta] \\ a \text { monic }}} \frac{1}{a^{n}} \in \mathbb{F}_{p}((1 / \theta)) \quad \text { ( } n \text { a positive integer }\right),
$$

which are the Carlitz zeta values associated to $\mathbb{F}_{p^{r}}[\theta]$. 
Writing down a $p^{r}$-adic expansion $\sum_{i} n_{r i} p^{r i}$ of $n$, we let

$$
\Gamma_{r(n+1)}:=\prod_{i=0}^{\infty} D_{r i}^{n_{r i}} .
$$

We call $\Gamma_{r(n+1)}$ the Carlitz factorials associated to $\mathbb{E}_{p^{r}}[\theta]$. The Bernoulli-Carlitz numbers $B_{r n}$ in $\mathbb{F}_{p}(\theta)$ are given by the following expansions from the Carlitz exponential series

$$
\frac{z}{\exp _{C_{r}}(z)}=\sum_{n=0}^{\infty} \frac{B_{r n}}{\Gamma_{r(n+1)}} z^{n} .
$$

We state the Euler-Carlitz relations:

Theorem 4.4.2 [Carlitz 1935]. For all positive integers $n$ divisible by $p^{r}-1$, one has

$$
\zeta_{r}(n)=\frac{B_{r n}}{\Gamma_{r(n+1)}} \tilde{\pi}_{r}^{n} .
$$

We call a positive integer $n(p, r)$-even if it is divisible by $p^{r}-1$; otherwise we call it $(p, r)$-odd. Thus, when $p=2$ and $r=1$, all positive integers are even.

4.4.3. The Anderson-Thakur formula. Anderson and Thakur [1990] introduced the $n$-th tensor power of the Carlitz $\mathbb{F}_{p^{r}}[t]$-module $C_{r}$, and they related $\zeta_{r}(n)$ to the last coordinate of the logarithm associated to the $n$-th tensor power of $C_{r}$ for each positive integer $n$. More precisely, they interpreted $\zeta_{r}(n)$ as $\mathbb{F}_{p^{r}}(\theta)$-linear combinations of $n$-th Carlitz polylogarithms of algebraic numbers:

Theorem 4.4.4 [Anderson and Thakur 1990]. Given any positive integers $r$ and $n$, one can find a sequence $h_{0, r n}, \ldots, h_{l_{r n}, r n} \in \mathbb{F}_{p^{r}}(\theta), l_{r n}<n p^{r} /\left(p^{r}-1\right)$, such that

$$
\zeta_{r}(n)=\sum_{i=0}^{l_{r n}} h_{i, r n} \operatorname{Plog}_{r n}\left(\theta^{i}\right),
$$

where $\operatorname{Plog}_{r n}(z)$ is defined as in (7). In the special case of $n \leq p^{r}-1$,

$$
\zeta_{r}(n)=\operatorname{Plog}_{r n}(1) .
$$

Definition 4.4.5. Given any positive integer $r$, for each $n \in \mathbb{N}$, $\left(p^{r}-1\right) \nmid n$, with $l_{r n}$ as given by (12), we fix a finite subset

$$
\left\{\alpha_{0, r n}, \ldots, \alpha_{m_{r n}, r n}\right\} \subseteq\left\{1, \theta, \ldots, \theta^{l_{r n}}\right\}
$$

such that

$$
\left\{\tilde{\pi}_{r}^{n}, \mathscr{L}_{0, r n}(\theta), \ldots, \mathscr{L}_{m_{r n}, r n}(\theta)\right\} \quad \text { and } \quad\left\{\tilde{\pi}_{r}^{n}, \zeta_{r}(n), \mathscr{L}_{1, r n}(\theta), \ldots, \mathscr{L}_{m_{r n}, r n}(\theta)\right\}
$$

are $\mathbb{F}_{p^{r}}(\theta)$-bases for $N_{r n}$, where $\mathscr{L}_{j, r n}(t):=L_{\alpha_{j}, r n}(t)$ for $j=0, \ldots, m_{r n}$. This can be done because of (12) (see [Chang and Yu 2007, § 4.1]), and note that $m_{r n}+2$ 
is the dimension of $N_{r n}$ over $\mathbb{F}_{p^{r}}(\theta)$. In the case of $p=2$ and $r=1$, the $\mathbb{F}_{p}(\theta)$ dimension of $N_{11}$ is 1 and we set $m_{11}:=-1$.

Definition 4.4.6. Given any positive integers $s$ and $d$ with $d \geq 2$, for each $1 \leq r \leq d$, we define

$$
\begin{cases}U_{r}(s)=\{1\} & \text { if } p=2 \text { and } r=1 ; \\ U_{r}(s)=\left\{1 \leq n \leq s ; p \nmid n,\left(p^{r}-1\right) \nmid n\right\} & \text { otherwise. }\end{cases}
$$

For each $n \in U_{r}(s)$, we define that if $p=2$ and $r=1$,

$$
\begin{aligned}
& \Phi_{r n}:=(t-\theta) \in \mathrm{GL}_{1}(\bar{k}(t)), \\
& \Psi_{r n}:=\Omega_{1} \in \mathrm{GL}_{1}(\mathbb{L}),
\end{aligned}
$$

otherwise

$$
\begin{aligned}
& \Phi_{r n}:=\Phi_{r n}\left(\alpha_{0, r n}, \ldots, \alpha_{m_{r n}, r n}\right) \in \mathrm{GL}_{\left(m_{r n}+2\right)}(\bar{k}(t)), \\
& \Psi_{r n}:=\Psi_{r n}\left(\alpha_{0, r n}, \ldots, \alpha_{m_{r n}, r n}\right) \in \mathrm{GL}_{\left(m_{r n}+2\right)}(\mathbb{L}) .
\end{aligned}
$$

By Theorem 4.3.1, we have that

$$
\operatorname{dim} \Gamma_{\Psi_{r n}}=\operatorname{tr} . \operatorname{deg}_{\bar{k}(t)} \bar{k}(t)\left(\Psi_{r n}\right)=m_{r n}+2 .
$$

Put $\Phi_{r}:=\bigoplus_{n \in U_{r}(s)} \Phi_{r n}$; then $\Phi_{r}$ defines a rigid analytically trivial pre- $t$-motive $M_{r}$ of level $r$ with rigid analytic trivialization $\Psi_{r}:=\bigoplus_{n \in U_{r}(s)} \Psi_{r n}$ (see (10)). Also, $M_{r}$ is of type $\mathbf{S V}$, and by Theorem 2.2.3, $M_{r}$ has the GP property. The main theorem of [Chang and Yu 2007] is the following:

Theorem 4.4.7 [Chang and $\mathrm{Yu}$ 2007, Theorem 4.5]. For any positive integers $s$ and $r$, the Galois group $\Gamma_{M_{r}}$ over $\mathbb{F}_{p^{r}}(t)$ is full, that is,

$$
\operatorname{dim} \Gamma_{M_{r}}=1+\sum_{n \in U_{r}(s)}\left(m_{r n}+1\right) .
$$

4.5. Proof of Theorem 1.2.1. Given any integer $d \geq 2$, we put $\ell:=\operatorname{lcm}(1, \ldots, d)$ and $\ell_{r}:=\ell / r$ for $r=1, \ldots, d$. For each $1 \leq r \leq \bar{d}$, let $\mathbf{M}_{r}:=M_{r}^{\left(\ell_{r}\right)}$ be the $\ell_{r}$-th derived pre-t $t$-motive of $M_{r}$ defined as above. Note that $\mathbf{M}_{r}$ is a rigid analytically trivial pre- $t$-motive of level $\ell$ that is still of type SV. By Proposition 2.2.2, each $\mathbf{M}_{r}$ has the GP property, and by Theorem 4.4.7, its Galois group $\Gamma_{\mathbf{M}_{r}}$ is full. Further, for each $1 \leq r \leq d$ any diagonal of $\mathbf{M}_{r}$ has canonical rigid analytic trivialization given by $\Omega_{r}^{n}$ for some $n \in U_{r}(s)$, and hence its Galois group is $\mathbb{G}_{m}$ because $\Omega_{r}$ is transcendental over $\bar{k}(t)$.

Since by Lemma 4.2.1 the functions $\Omega_{1}, \ldots, \Omega_{d}$ are algebraically independent over $\bar{k}(t)$, particularly the Galois group of $N_{i} \oplus N_{j}$ is a two-dimensional torus over $\mathbb{F}_{p^{\ell}}(t)$ for any diagonal $N_{i}$ (resp. $N_{j}$ ) of $\mathbf{M}_{i}\left(\operatorname{resp} . \mathbf{M}_{j}\right.$ ) with $i \neq j, 1 \leq i, j \leq d$. Put $\mathbf{M}:=\bigoplus_{r=1}^{d} \mathbf{M}_{r}$, and note that $\mathbf{M}$ has the GP property by Corollary 2.2.4. Applying Theorem 3.2.2 to this situation, we obtain the explicit dimension of $\Gamma_{\mathbf{M}}$ : 
Theorem 4.5.1. Given any positive integers $s$ and $d$ with $d \geq 2$, let $\mathbf{M}$ be defined as above. Then the Galois group $\Gamma_{\mathbf{M}}$ is full, that is,

$$
\operatorname{dim} \Gamma_{\mathbf{M}}=d+\sum_{r=1}^{d}\left(m_{r n}+1\right) .
$$

As a consequence, we completely determine all the algebraic relations among the families of Carlitz zeta values:

Corollary 4.5.2. Given any positive integers $d$ and $s$, the transcendence degree of the field

$$
\bar{k}\left(\bigcup_{r=1}^{d}\left\{\tilde{\pi}_{r}, \zeta_{r}(1), \ldots, \zeta_{r}(s)\right\}\right)
$$

over $\bar{k}$ is

$$
\sum_{r=1}^{d}\left(s-\left\lfloor\frac{s}{p}\right\rfloor-\left\lfloor\frac{s}{p^{r}-1}\right\rfloor+\left\lfloor\frac{s}{p\left(p^{r}-1\right)}\right\rfloor+1\right) .
$$

Proof. We may assume $d \geq 2$, since the case $d=1$ is already given in [Chang and Yu 2007, Corollary 4.6]. For $1 \leq r \leq d$, let

$$
\begin{cases}V_{1}(s):=\varnothing & \text { if } p=2 \\ V_{r}(s):=U_{r}(s) & \text { otherwise. }\end{cases}
$$

Since $\mathbf{M}$ has the GP property, by Theorem 4.5.1 we see that the elements of the set

$$
\left\{\Omega_{1}(\theta), \ldots, \Omega_{d}(\theta)\right\} \cup\left(\bigcup_{r=1}^{d} \bigcup_{n \in V_{r}(s)}\left\{\mathscr{L}_{0, r n}(\theta), \ldots, \mathscr{L}_{m_{r n}, r n}(\theta)\right\}\right)
$$

are algebraically independent over $\bar{k}$. In particular, by Definition 4.4 .5 , we have that

$$
\left\{\tilde{\pi}_{1}, \ldots, \tilde{\pi}_{d}\right\} \cup\left(\bigcup_{r=1}^{d} \bigcup_{n \in V_{r}(s)}\left\{\zeta_{r}(n)\right\}\right)
$$

is an algebraically independent set over $\bar{k}$. Counting the cardinality of $V_{r}(s)$ for each $1 \leq r \leq d$, we complete the proof.

\section{Acknowledgements}

We thank D. Thakur for many helpful discussions during the preparation of this manuscript. The first author thanks the NSC and National Center for Theoretical Sciences for financial support, and Texas A\&M University for its hospitality.

\section{References}

[Anderson 1986] G. W. Anderson, “t-motives”, Duke Math. J. 53:2 (1986), 457-502. MR 87j:11042 
[Anderson and Thakur 1990] G. W. Anderson and D. S. Thakur, "Tensor powers of the Carlitz module and zeta values", Ann. of Math. (2) 132:1 (1990), 159-191. MR 91h:11046 Zbl 0713.11082

[Anderson et al. 2004] G. W. Anderson, W. D. Brownawell, and M. A. Papanikolas, "Determination of the algebraic relations among special $\Gamma$-values in positive characteristic", Ann. of Math. (2) 160:1 (2004), 237-313. MR 2005m:11140 Zbl 1064.11055

[Carlitz 1935] L. Carlitz, "On certain functions connected with polynomials in a Galois field", Duke Math. J. 1:2 (1935), 137-168. MR 1545872 Zbl 0012.04904

[Chang 2009] C.-Y. Chang, "A note on a refined version of Anderson-Brownawell-Papanikolas criterion”, J. Number Theory 129:3 (2009), 729-738. MR 2010a:11144 Zbl 1187.11022

[Chang and Yu 2007] C.-Y. Chang and J. Yu, "Determination of algebraic relations among special zeta values in positive characteristic", Adv. Math. 216:1 (2007), 321-345. MR 2008j:11114 Zbl 1123.11025

[Chang et al. 2010] C.-Y. Chang, M. A. Papanikolas, D. S. Thakur, and J. Yu, "Algebraic independence of arithmetic gamma values and Carlitz zeta values", Adv. Math. 223:4 (2010), 1137-1154. MR 2011d:11180 Zbl 05671646

[Denis 1998] L. Denis, "Indépendance algébrique de différents $\pi$ ", C. R. Acad. Sci. Paris Sér. I Math. 327:8 (1998), 711-714. MR 99i:11059 Zbl 0922.11055

[Goss 1996] D. Goss, Basic structures of function field arithmetic, Ergebnisse der Mathematik und ihrer Grenzgebiete (3) 35, Springer, Berlin, 1996. MR 97i:11062 Zbl 0874.11004

[Papanikolas 2008] M. A. Papanikolas, "Tannakian duality for Anderson-Drinfeld motives and algebraic independence of Carlitz logarithms", Invent. Math. 171:1 (2008), 123-174. MR 2009b:11127 Zbl 05236753

[Thakur 2004] D. S. Thakur, Function field arithmetic, World Scientific, River Edge, NJ, 2004. MR 2005h:11115 Zbl 1061.11001

[Wade 1941] L. I. Wade, "Certain quantities transcendental over $\operatorname{GF}\left(p^{n}, x\right)$ ”, Duke Math. J. 8 (1941), 701-720. MR 3,263f Zbl 0063.08101

[Yu 1991] J. Yu, "Transcendence and special zeta values in characteristic p", Ann. of Math. (2) 134:1 (1991), 1-23. MR 92g:11075 Zbl 0734.11040

[Yu 1997] J. Yu, "Analytic homomorphisms into Drinfeld modules", Ann. of Math. (2) 145:2 (1997), 215-233. MR 98c:11054 Zbl 0881.11055

Communicated by Brian Conrad

Received 2010-01-27 Revised 2010-10-18 Accepted 2010-11-21

cychang@math.cts.nthu.edu.tw National Center for Theoretical Sciences,

Mathematics Division, National Tsing Hua University,

Hsinchu City 30042, Taiwan

http://math.cts.nthu.edu.tw/ cychang/

map@math.tamu.edu

Department of Mathematics, Texas A\&M University, College Station, TX 77843-3368, United States http://www.math.tamu.edu/ map/

yu@math.ntu.edu.tw

Department of Mathematics, National Taiwan University, Taipei City 106, Taiwan

http://www.math.ntu.edu.tw/ yu/ 


\section{Algebra \& Number Theory}

www.jant.org

\section{EDITORS}

MANAGING EDITOR

Bjorn Poonen

Massachusetts Institute of Technology

Cambridge, USA

\author{
EDITORIAL BOARD CHAIR \\ David Eisenbud \\ University of California \\ Berkeley, USA
}

BOARD OF EDITORS

Georgia Benkart

Dave Benson

Richard E. Borcherds

John H. Coates

J-L. Colliot-Thélène

Brian D. Conrad

Hélène Esnault

Hubert Flenner

Edward Frenkel

Andrew Granville

Joseph Gubeladze

Ehud Hrushovski

Craig Huneke

Mikhail Kapranov

Yujiro Kawamata

János Kollár

Yuri Manin

Barry Mazur

Susan Montgomery
University of Wisconsin, Madison, USA

University of Aberdeen, Scotland

University of California, Berkeley, USA

University of Cambridge, UK

CNRS, Université Paris-Sud, France

University of Michigan, USA

Universität Duisburg-Essen, Germany

Ruhr-Universität, Germany

University of California, Berkeley, USA

Université de Montréal, Canada

San Francisco State University, USA

Hebrew University, Israel

University of Kansas, USA

Yale University, USA

University of Tokyo, Japan

Princeton University, USA

Northwestern University, USA

Harvard University, USA

University of Southern California, USA
Shigefumi Mori

Andrei Okounkov

Raman Parimala

Victor Reiner

Karl Rubin

Peter Sarnak

Michael Singer

Ronald Solomon

Vasudevan Srinivas

J. Toby Stafford

Bernd Sturmfels

Richard Taylor

Ravi Vakil

Michel van den Bergh

Marie-France Vignéras

Kei-Ichi Watanabe

Andrei Zelevinsky

Efim Zelmanov
RIMS, Kyoto University, Japan

Princeton University, USA

Emory University, USA

University of Minnesota, USA

University of California, Irvine, USA

Princeton University, USA

North Carolina State University, USA

Ohio State University, USA

Tata Inst. of Fund. Research, India

University of Michigan, USA

University of California, Berkeley, USA

Harvard University, USA

Stanford University, USA

Hasselt University, Belgium

Université Paris VII, France

Nihon University, Japan

Northeastern University, USA

University of California, San Diego, USA

\section{PRODUCTION}

contact@msp.org

Silvio Levy, Scientific Editor

Andrew Levy, Production Editor

See inside back cover or www.jant.org for submission instructions.

The subscription price for 2011 is US \$150/year for the electronic version, and \$210/year (+ \$35 shipping outside the US) for print and electronic. Subscriptions, requests for back issues from the last three years and changes of subscribers address should be sent to Mathematical Sciences Publishers, Department of Mathematics, University of California, Berkeley, CA 94720-3840, USA.

Algebra \& Number Theory (ISSN 1937-0652) at Mathematical Sciences Publishers, Department of Mathematics, University of California, Berkeley, CA 94720-3840 is published continuously online. Periodical rate postage paid at Berkeley, CA 94704, and additional mailing offices.

ANT peer review and production are managed by EditFLOw ${ }^{\mathrm{TM}}$ from Mathematical Sciences Publishers.

PUBLISHED BY

mathematical sciences publishers

http://msp.org/

A NON-PROFIT CORPORATION

Typeset in LATEX

Copyright $\odot 2011$ by Mathematical Sciences Publishers 


\section{Algebra \& Number Theory}

\section{Volume $5 \quad$ No. $1 \quad 2011$}

Formules pour l'invariant de Rost

PhILIPPE GILle and ANNE QUÉGUINER-MATHIEU

Modular abelian varieties of odd modular degree

SOROOSH YAZDANI

Group algebra extensions of depth one

ROBERT BOLTJE and BURKHARD KÜLSHAMMER

Set-theoretic defining equations of the variety of principal minors of symmetric matrices

LUKE OEDING

Frobenius difference equations and algebraic independence of zeta values in positive equal characteristic

Chieh-Yu Chang, Matthew A. Papanikolas and Jing Yu 